0.0001 and $r=0.728, p<0.0001$, respectively). ACPA and RF positive patients did not have higher FRAX scores (including or not BMA). Patients with erosive disease had a higher 10-year probability of major fracture evaluated by FRAX when it includes BMD $(p=0.041)$.

Conclusion: It is very important to accurately assess the risk of osteoporotic fractures in RA patients to treat them properly. The authors highlight the high number of patients who are not receiving treatment according to FRAX categorization. In spite of the correlation between estimated fracture risk by FRAX with and without BMD, there is a discordance in fracture risk categorization, as one fifth of patients of low risk were reclassified as high risk. For the RA population treated with bDMARDS, our findings raise the need to request a DXA not only for patients classified as having an intermediate risk of fracture, but also for lowrisk patients.

Disclosure of Interests: Maria Rato: None declared, Filipe Pinheiro: None declared, Salomé Garcia: None declared, Bruno Miguel Fernandes: None declared, Sara Ganhão: None declared, Rita Gaio: None declared, Miguel Bernardes Speakers bureau: Abbvie, Amgen, Biogen, Eli-Lilly, Glaxo-Smith-Kline, Pfizer, Janssen, Novartis, Alexandra Bernardo: None declared, Lúcia Costa: None declared

DOI: 10.1136/annrheumdis-2020-eular.3354

\begin{tabular}{l|l}
\hline SAT0483 & SAFETY OF INTRAVENOUS IBANDRONIC ACID \\
IN CHRONIC KIDNEY DISEASE: A REAL WORLD \\
EXPERIENCE
\end{tabular}

J. Southern ${ }^{1}$, M. Chakravorty ${ }^{1}$, L. H. Lee ${ }^{1} .{ }^{1}$ Royal Derby Hospital, Derby, United Kingdom

Background: Common forms of intravenous bisphosphonate used at the Royal Derby Hospital are zoledronic acid and ibandronic acid for a variety of indications. In the treatment of osteoporosis, zoledronic acid is preferred due to its convenience of once-yearly dosing; compared to ibandronic acid which is given three-monthly. Zoledronic acid is contraindicated in patients with an estimated glomerular filtration rate (eGFR) of less than 35 due to nephrotoxicity concerns. Ibandronic acid, however, is generally offered with an eGFR of 30 or over and is perceived to be a safer choice in more advanced chronic kidney disease. The potential of extending the use of ibandronic acid to patients with lower eGFR is being explored. However, there is a paucity of real world data and this study will therefore seek to affirm the safety profile in those on treatment.

Objectives: Establish the safety profile of IV ibandronic acid with regards to worsening renal function or significant hypocalcaemia injury in the context of reduced renal clearance.

Methods: The details of patients receiving IV ibandronic acid at Royal Derby Hospital were retrieved from the osteoporosis department register in September 2019. Data was collected anonymously from records using the electronic prescribing and pathology hospital database, together with electronic letters. The first three pre-infusion serum adjusted calcium levels, vitamin D, creatinine and eGFR were recorded. In addition, results from initiation to present were screened for any episodes of hypocalcaemia, acute kidney injury (AKI) or significant decline in renal function.

Results: Treatment duration ranged from 6 months to 6 years. Female:male ratio was 9:1 and the average age was 75 years (range 50-90). Baseline eGFR ranged from 27 to over $60 ; 3$ patients had eGFR $\geq 60,2$ had eGFR 27 while remaining patients (75\%) had eGFR $30-59$. All patients received a standard $3 \mathrm{mg}$ infusion on each occasion. The most common rationale cited for ibandronic acid choice as opposed to zoledonic acid was reduced creatinine clearance or eGFR. Three patients (15\%) developed one or more episodes of mild hypocalcaemia (lowest $2.01 \mathrm{mmol} / \mathrm{l}$ ). No episodes of hypocalcaemia were identified in the first three pre-infusion levels. Four patients $(25 \%)$ had a decline in eGFR by more than $5 \mathrm{ml} / \mathrm{min} / 1.73 \mathrm{~m}^{2}$ but there was no definitive causal link with ibandronic acid and was most commonly felt to be related to their underlying renal disease. Three patients $(15 \%)$ had at least one episode of AKI since commencing treatment, each explained by an intercurrent illness. Serum Vitamin D levels were measured pre-infusion in $92 \%$ of cases.

Conclusion: This study reaffirms the safety profile of ibandronic acid use in renal function as low as CKD Stage $3 b\left(\geq 30 \mathrm{ml} / \mathrm{min} / 1.73 \mathrm{~m}^{2}\right)$. No episodes of $A K I$ or sustained decline in renal function were causally linked to ibandronic acid. References: Royal Derby Hospital Proposed Clinical Guideline (2019) - Use of ibandronic acid in CKD 4 at reduced dosage.

Disclosure of Interests: None declared

DOI: 10.1136/annrheumdis-2020-eular.840

\section{SAT0484 \\ TRABECULAR BONE SCORE IN SYSTEMIC LUPUS ERYTHEMATOSUS PATIENTS}

A. Casabella ${ }^{1}$, S. Paolino ${ }^{1}$, E. Alessandri ${ }^{1}$, V. Smith ${ }^{2}$, B. Ruaro ${ }^{3}$, C. Pizzorni ${ }^{1}$, A. Sulli ${ }^{1}$, M. Cutolo ${ }^{1} .{ }^{1}$ Genoa, Research Laboratory and Academic Division of Rheumatology, Department of Internal Medicine, University of Genova, IRCCS San Martino Polyclinic Hospital, Genoa, Italy; ${ }^{2}$ Ghent, Department of Rheumatology, Ghent University Hospital, Department of Internal Medicine, Ghent UniversityUnit for Molecular Immunology and Inflammation, VIB Inflammation Research Center (IRC); Ghent Belgium, Ghent, Belgium; ${ }^{3}$ Parma, Cardinal Ferrari Center, S. Stefano Rehabilitation, Fontanellato-Parma, Italy, Parma, Italy

Background: Systemic lupus erythematosus (SLE) patients shown an increased risk of low bone mass as a result of multifactorial events: physical inactivity, persistent inflammation, low vitamin D levels (photosensitivity) and glucocorticoid treatment. Trabecular Bone Score (TBS), is an index extracted from the dual-energy X-ray absorptiometry (DXA) that provides an indirect measurement of bone axial microarchitecture and allows to get information about bone quality in several rheumatic diseases (1-4)

Objectives: The aims of this study were to examine the prevalence and risk factors for low bone mineral density (BMD) (osteoporosis or osteopenia) in female patients affected by SLE and to compare with matched healthy subjects (CNT). Methods: 70 female patients (mean age $41 \pm 20$ years) affected by SLE and 65 age- matched CNT (mean age $46 \pm 7$ years) were enrolled. Bone Mineral Density (BMD, g/cm2) of the lumbar spine (L1-L4) was analyzed using a DXA scan (GE Lunar Prodigy). Lumbar spine TBS was derived for each spine DXA examination using the TBS index (TBS iNsight Medimaps).

Results: The mean $B M D \pm S D$ was $0.47 \pm 0.57 \mathrm{~g} / \mathrm{cm} 2$ at the lumbar spine and $0.78 \pm 0.22 \mathrm{~g} / \mathrm{cm} 2$ at the hip in SLE patients. The prevalence of osteopenia was $40.0 \%$ and was $19.4 \%$ of osteoporosis in SLE patients. Most of SLE patients (75\%) presented a bone loss that was significantly higher when compared with control group $(p<0.001)$. Lumbar spine TBS score was found significantly lower in SLE patients compared with CNT $(0.687 \pm 0.675$ vs, $1.294 \pm 0.809 p<0.001$ respectively) and of $0,47 \pm 0,94$ times lower than expected from the concomitan reference BMD value.

Conclusion: The study shows that the further TBS analysis, independently from the concomitant BMD value, is significatively lower then expected in SLE patients. The detection of the TBS, together with the BMD, may offer a more reliable indication of the real whole bone condition in chronic and systemic inflammatory rheumatic diseases, such as SLE.

References:

[1] Cutolo $M$ et al. Ann Rheum Dis. 2009;68 446-7; 2 Dey M et al. Lupus. 018;271547-1551; 3 Ruaro B, Casabella A, et al. Rheumatology (Oxford) 2018;57:1548-1554. 4 Ruaro B, Casabella A, et al. Clin Rheumatol. 2018 Nov;37(11):3057-3062.

Disclosure of Interests: Andrea Casabella: None declared, Sabrina Paolino: None declared, Elisa Alessandri: None declared, Vanessa Smith Grant/ research support from: The affiliated company received grants from Research Foundation - Flanders (FWO), Belgian Fund for Scientific Research in Rheumatic diseases (FWRO), Boehringer Ingelheim Pharma $\mathrm{GmbH}$ \& $\mathrm{Co}$ and Janssen-Cilag NV, Consultant of: Boehringer-Ingelheim Pharma GmbH \& Co, Speakers bureau: Actelion Pharmaceuticals Ltd, Boehringer-Ingelheim Pharma $\mathrm{GmbH} \&$ Co and UCB Biopharma Sprl, Barbara Ruaro: None declared, Carmen Pizzorni: None declared, Alberto Sulli Grant/research support from: Laboratori Baldacci, Maurizio Cutolo Grant/research support from: Bristol-Myers Squibb, Actelion, Celgene, Consultant of: Bristol-Myers Squibb, Speakers bureau: Sigma-Alpha DOI: 10.1136/annrheumdis-2020-eular.4180

\section{SAT0485 \\ PERCENTAGE BODY FAT HAS A STRONGER ASSOCIATION WITH BONE MINERAL DENSITY AT THE HIP AND SPINE COMPARED TO BODY MASS INDEX}

C. Thurston ${ }^{1}$, R. Tribbick ${ }^{2}$, J. Kerns ${ }^{1}$, F. Dondelinger ${ }^{2}$, M. Bukhari ${ }^{1,3} .{ }^{1}$ Lancaster University, Lancaster Medical School, Lancaster, United Kingdom; ${ }^{2}$ Lancaster University, CHICAS, Lancaster, United Kingdom; ${ }^{3}$ University Hospitals of Morecambe Bay NHS Foundation Trust, Lancaster, United Kingdom

Background: A decreased body mass index (BMI) is associated with poorer bone health, a decreased bone mineral density (BMD), and an increased fracture risk. Cardiovascular (CVS) data has shown that the waist:hip ratio is a more robust measurement for CVS outcomes than BMI (1). Waist:hip ratio has never been evaluated as an outcome measure for bone health. Dual-energy $x$-ray absorptiometry (DEXA) has the capacity to measure average percentage fat in the L1-L4 region and at the hip, and directly relates to the measurement of waist:hip ratio.

Objectives: To evaluate the relationship between BMD and average percent fat in a cohort referred for DEXA scanning.

Methods: We analysed data routinely collected from patients referred for DEXA between 2004 and 2010 at the Royal Lancaster Infirmary in the North of England. Data collected for these patients included DEXA scans 